\title{
Late durability of decellularized allografts for aortic valve replacement: A word of caution
}

\author{
Meghana R. K. Helder, MD, ${ }^{a}$ Nicholas T. Kouchoukos, MD, ${ }^{b}$ Kenton Zehr, MD, ${ }^{c}$ Joseph A. Dearani, MD, ${ }^{a}$ \\ Joseph J. Maleszewski, MD, ${ }^{\mathrm{d}}$ Charles Leduc, MD ${ }^{\mathrm{d}}$ Courtney N. Heins, BS, ${ }^{\mathrm{e}}$ and Hartzell V. Schaff, MD, ${ }^{\mathrm{a}}$ \\ Rochester, Minn; St Louis, Mo; and Baltimore, Md
}

\footnotetext{
From the Divisions of a Cardiovascular Surgery and ${ }^{\mathrm{d}}$ Anatomic Pathology, Mayo Clinic; ${ }^{\mathrm{b}}$ Missouri Baptist Medical Center, St Louis, Mo; ${ }^{\mathrm{C}}$ Heart and Vascular Institute, Johns Hopkins Medicine, Baltimore, Md; and ${ }^{\mathrm{e}}$ Department of Biomedical Statistics and Informatics, Mayo Clinic, Rochester, Minn.

Disclosures: Authors have nothing to disclose with regard to commercial support.

Presented at the Eleventh Annual Academic Surgical Congress, Jacksonville, Fla, February 4, 2016.

Received for publication Feb 5, 2016; revisions received March 4, 2016; accepted for publication March 16, 2016; available ahead of print April 28, 2016.

Address for reprints: Hartzell V. Schaff, MD, Division of Cardiothoracic Surgery, Joseph 5-200, St Mary's Hospital, Mayo Clinic, 1216 Second St SW, Rochester, MN 55902 (E-mail: schaff.hartzell@mayo.edu).

J Thorac Cardiovasc Surg 2016;152:1197-9 $0022-5223 / \$ 36.00$

Copyright (C) 2016 by The American Association for Thoracic Surgery http://dx.doi.org/10.1016/j.jtcvs.2016.03.050
}

Early outcomes of decellularized allografting (DAVA) performed for aortic root replacement (ARR) have been promising. Da Costa and colleagues ${ }^{1}$ reported only 1 reoperation among 38 patients, but follow-up was relatively short (19 months). Assessment of the durability of DAVA requires long-term follow-up.

\section{METHODS}

After receiving approval by the appropriate Institutional Review Boards, we reviewed patients who underwent ARR with DAVA between March 12, 2002, and October 6, 2004, at Mayo Clinic, Rochester, Minnesota and Missouri Baptist Medical Center, St Louis, Missouri. We compared outcomes between those patients and patients who underwent ARR with a standard cryopreserved allograft during the same period. The primary outcome was reoperation, and the secondary outcome was survival.

\section{Pathologic Analysis}

Sections of excised valve and cusp tissue from the DAVA were stained with hematoxylin and eosin and Verhoeff-Van Gieson stains. Graft analysis (performed by J.J.M. and C.L.) involved grading of inflammation, fibrosis, calcification, recellularization, and degeneration.

\section{Statistical Analysis}

Descriptive statistics for categorical variables are reported as frequency and percentage, and continuous variables are reported as mean \pm standard deviation or median (range). The Kaplan-Meier method was used to calculate survival and freedom of reoperation estimates. All statistical tests were 2 -sided, with the $\alpha$ level set at 0.05 for statistical significance.

\section{RESULTS}

Forty-two patients ( $74 \%$ men; mean age, $49 \pm 17$ years) underwent ARR with a DAVA. Aortic valve reoperation was required in $37 \%$ of the survivors ( 15 of 41 ). Indications were endocarditis ( $26 \% ; n=11$ of 42$)$, aortic valve regurgitation associated with aortic aneurysm $(12 \% ; n=5)$, isolated

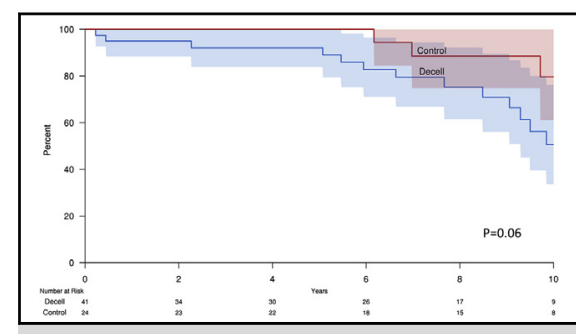

Kaplan-meier curves for freedom from reoperation Curves vary significantly at 10 years $(P=.06)$.

Central Message
Patients receiving a decellularized aortic allo-
graft had a freedom from reoperation at 10 years
of $51 \%(95 \% \mathrm{CI}, 34 \%-76 \%)$, compared with
$80 \%(95 \% \mathrm{CI}, 60 \%-100 \%)$ in those who
received a standard cryopreserved allograft
$(P=.06)$.

See Article page 1156.

See Editorial Commentaries page 1165 1200 , and 1202 .

aortic valve regurgitation $(31 \% ; \mathrm{n}=13)$, aortic valve stenosis $(29 \% ; \mathrm{n}=12)$, and aortic dissection causing aortic valve regurgitation $(2 \% ; n=1)$. Twenty-nine patients underwent ARR with a cryopreserved allograft. Indications for operation in these 29 patients included endocarditis $(21 \%$; $\mathrm{n}=6)$, aortic aneurysm $(10 \% ; \mathrm{n}=3)$, aortic valve regurgitation $(14 \% ; n=4)$, aortic valve stenosis $(55 \% ; n=16)$. The prevalence of preoperative endocarditis in this group was similar to that in the group of patients who received DAVA $(P=.20)$. Aortic valve reoperation was required in 4 of the 24 control patients $(17 \%)$, including 2 patients with regurgitation and 2 with stenosis.

Of the 10 valves with sufficient tissue for analysis, 7 had edematous degeneration and calcification of the valve. Mild recellularization was present in the other 3 valves. Adventitial fibrosis and neointimal fibroplasia were identified in all cases. Fibrosis and calcification have been reported as modes of failure in cryopreserved allografts (Figure 1).

Freedom from reoperation at 5 years postsurgery was $92 \%(95 \%$ CI, $84 \%-100 \%)$ in the DAVA group and $100 \%$ in the control group. Freedom from reoperation at 


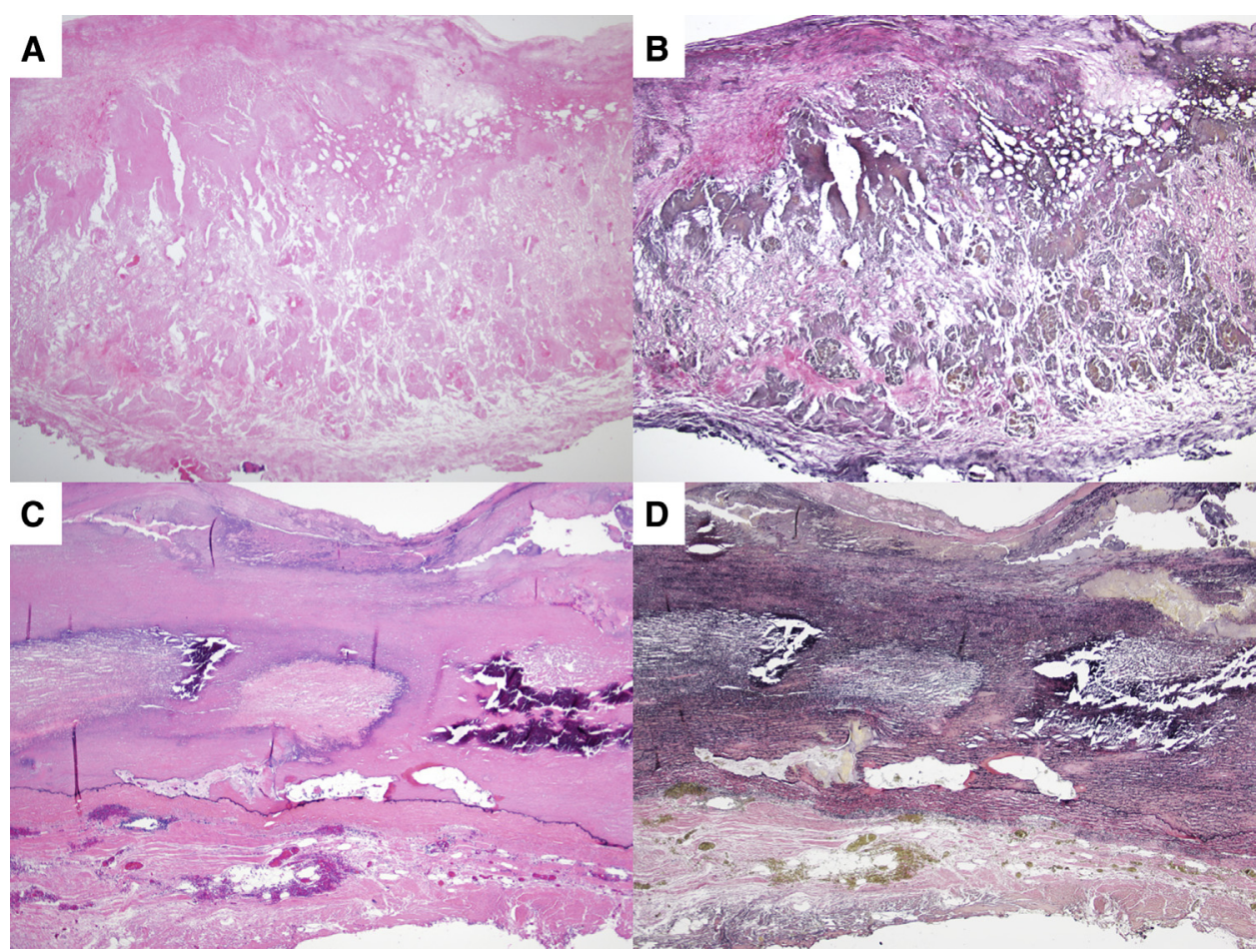

FIGURE 1. Histopathologic findings of DAVAs in tissue sections stained with hematoxylin and eosin (left column) and corresponding Verhoeff-Van Gieson elastic (right column) stains, showing (A and B) full-thickness degeneration of the valve cusp and (C and D) marked calcification of the tubular component of the graft. (Original magnification, $40 \times$.)

10 years postsurgery was $51 \%(95 \%$ CI, 34\%-76\%) in the DAVA group and $80 \%(95 \% \mathrm{CI}, 60 \%-100 \%)$ in the control group $(P=.06)$ (Figure 2). Overall 5- and 10 -year survival rates were $90 \%(95 \%$ CI, $80 \%-100 \%)$ and $76 \%(95 \% \mathrm{CI}, 61 \%-93 \%)$, respectively, in the DAVA group, compared with $72 \%$ (95\% CI, 57\%-90\%) and $57 \%(95 \% \mathrm{CI}, 38 \%-79 \%)$ in the control group $(P=.09)$.

\section{DISCUSSION}

Early outcomes in the patients undergoing ARR with DAVA were promising. A Brazilian study reported a

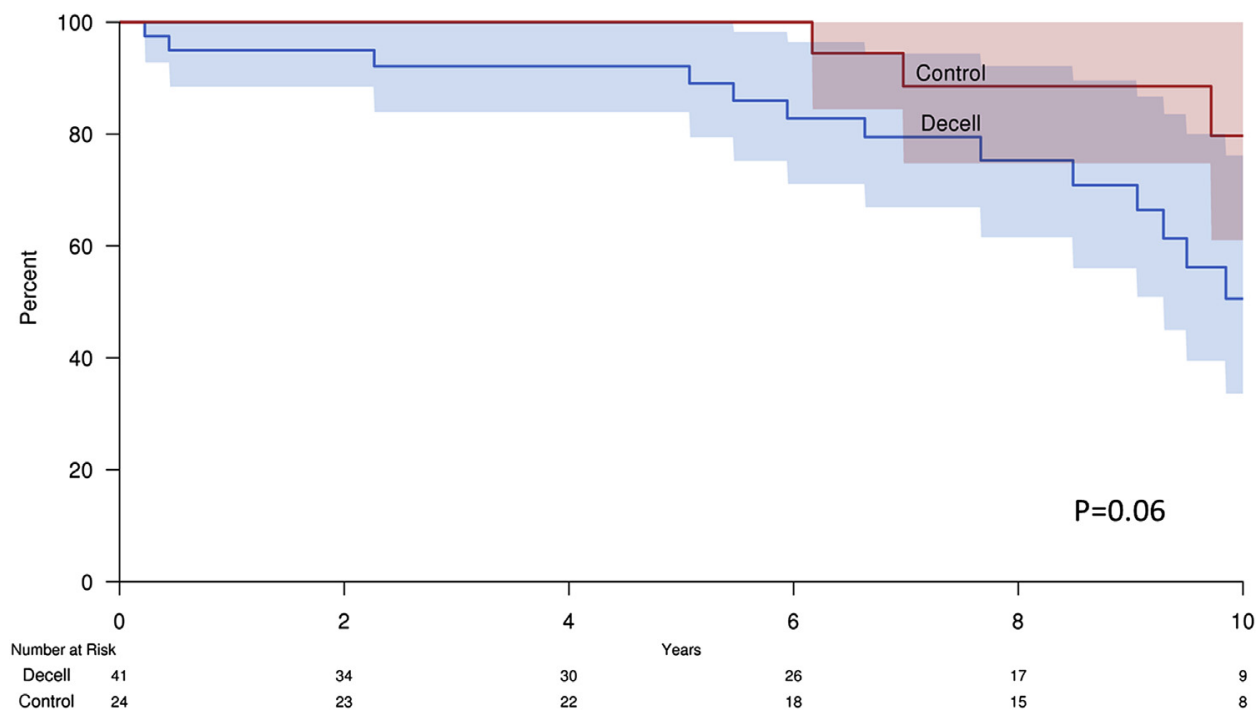

FIGURE 2. Kaplan-Meier curves for freedom from reoperation. Patients implanted with a DAVA had a $92 \%$ ( $95 \%$ CI, $84 \%-100 \%)$ freedom from reoperation at 5 years postimplantation, compared with $100 \%$ in the control group. Freedom from reoperation at 10 years postoperatively was $51 \%$ $(95 \% \mathrm{CI}, 34 \%-76 \%)$ in patients receiving a DAVA, compared with $80 \%(95 \% \mathrm{CI}, 60 \%-100 \%)$ in the control group $(P=.06)$. 
$98 \%$ freedom from reoperation at an average of 19 months of follow-up. ${ }^{1}$ In our study at 5 years, patients with DAVA had a $92 \%$ freedom from reoperation. Further, early hemodynamic performance appears to be favorable, with a mean aortic valve gradient of $8.8 \pm 6.3 \mathrm{~mm} \mathrm{Hg}$ in patients with DAVA assessed at an average of 30 months. ${ }^{2}$ Long-term outcomes are not favorable, however. At 10 years, freedom from reoperation in the patients receiving DAVA was $51 \%$, substantially lower than that in the patients receiving a standard cryopreserved allograft.

Based on the pathologic studies, modes of failure appear to be similar with DAVA and standard allografts. In explanted DAVAs, fibrosis and calcification were seen, with only minimal recellularization in the minority of cases. These findings likely reflect a final pathway of degeneration, but initiating mechanisms are unclear. There are several theoretical causes of the initial injury. First, the decellularization process may be damaging to tissue. $^{3}$ The decellularization process of the valves used in our study (CryoLife, Inc, Kennesaw, Ga) is different from that used in the study reported by da Costa and colleagues. ${ }^{1}$ Long-term follow-up studies from that group may be provide insight into whether the decellularization method affects allograft durability. Sterilization of decellularized valves could have a detrimental effect on durability as well. In an animal study, we demonstrated that sterilization with gamma radiation, even at doses as low as 3000 Gy, irreversibly damages decellularized porcine valves. ${ }^{4}$ The sterilization procedure for the allografts implanted in this study was proprietary, and strength testing after sterilization should be made available.

Long-term survival was generally similar in the 2 groups of patients. Overall 10-year survival was $76 \%$ in the DAVA group, compared with $57 \%$ in the control group $(P=.09)$. The decreased survival of control patients is likely due to their older age (7 years). Moreover, all of the DAVA implantations at one institution were performed as part of a study and as such, allografts were used in some patients who otherwise might not have received an allograft.

In conclusion, this study identified a strong trend for late reoperation in the DAVA group. Given the dramatic change in valve durability beyond 5 years, new tissue-engineered valves should be studied over the longer term before their use becomes widespread.

\section{References}

1. da Costa FD, Costa AC, Prestes R, Domanski AC, Balbi EM, Ferreira AD, et al. The early and midterm function of decellularized aortic valve allografts. Ann Thorac Surg. 2010;90:1854-60.

2. Zehr KJ, Yagubyan M, Connolly HM, Nelson SM, Schaff HV. Aortic roo replacement with a novel decellularized cryopreserved aortic homograft: postoperative immunoreactivity and early results. J Thorac Cardiovasc Surg. 2005; 130:1010-5

3. Liao J, Joyce EM, Sacks MS. Effects of decellularization on the mechanical and structural properties of the porcine aortic valve leaflet. Biomaterials. 2008;29: 1065-74.

4. Helder MR, Hennessy RS, Spoon DB, Tefft BJ, Witt TA, Marler RJ, et al Low-dose gamma irradiation of decellularized heart valves results in tissue injury in vitro and in vivo. Ann Thorac Surg. 2015;101:667-74. 